\title{
Téoros
}

Revue de recherche en tourisme

\section{Québec : planification, stratégie, marketing}

\section{Suzanne Chassé}

Volume 8, numéro 1, mars 1989

France-Québec

URI : https://id.erudit.org/iderudit/1080350ar

DOI : https://doi.org/10.7202/1080350ar

Aller au sommaire du numéro

\section{Éditeur(s)}

Université du Québec à Montréal

\section{ISSN}

0712-8657 (imprimé)

1923-2705 (numérique)

Découvrir la revue

Citer cet article

Chassé, S. (1989). Québec : planification, stratégie, marketing. Téoros, 8(1),

10-13. https://doi.org/10.7202/1080350ar d'utilisation que vous pouvez consulter en ligne.

https://apropos.erudit.org/fr/usagers/politique-dutilisation/ 


\section{Québec: \\ planification, stratégie, marketing}

L'industrie touristique est en constante évolution et la concurrence à l'échelle planétaire est de plus en plus féroce pour s'approprier une partie des centaines de milliards de dollars qu'elle génère. Les différents Etats tout comme les entreprises doivent s'adapter rapidement aux grandes tendanoes, aux changements structurels et aux événements conjoncturels.

Le défi consiste à performer dans cette industrie en mouvement perpétuel. Pour les gouvernements, le défi est énorme car ils ne sont pas propriétaires des entreprises. Ils ont d'abord un rôle de concertation des intervenants et par l'intermédiaire de programmes d'aide financière, ils peuvent encourager des initiatives du milieu correspondant aux orientations stratégiques que les intervenants du tourisme se sont fixes. Pour les entreprises, le défi est d'adapter l'offre à la demande car plus que jamais le consommateur est le maltre du jeu. Il faut que ces entrepreneurs fassent coïncider le produit aux exigences, aux nouvelles habitudes et aux nouvelles valeurs des consommateurs, autant que faire se peut.

Pour y arriver, les intervenants du tourisme québécois se sont done dotés d'une planification stratégique de marketing pour les prochaines années. Pour cela, une consultation a été réalisée auprès des différents intervenants, qu'ils soient représentants des associations touristiques régionales ou des associations sectorielles, chefs de file de l'entreprise privée, grossistes, transporteurs,... ou encore gestionnaires, chefs de service ou représentants en territoire du ministère du Tourisme du Québec.

Avant d'elaborer les strategies, les intervenants se sont entendus sur les menaces et opportunités de l'environnement du tourisme et sur un diagnostic identifiant les forces et faiblesses de la mise en marché du tourisme québécois. Suite à ces premiers constats, des objectifs tant qualitatifs que quantitatifs ont été proposés, des axes stratégiques ont été acoeptés et c'est par la suite qu'un plan stratégique d'ensemble a été élaboré. Il était essentiel que les intervenants soient d'accord sur le diagnostic (identification du problème) pour que les solutions avancés soient acceptées.

\section{Le contexte}

- Suzanne Chassé est enstignante au Collège de Granby et consultante en tourisme
Dans le contexte international, trois phénomènes majeurs interviennent afin de modifier la situation:

- la concurrence planétaire (l'obstacle distance $n^{\top} e n$ étant plus un);

- la continentalisation (Europe 92 - libre Echange USA-Canada, ...);

- la deréglementation aerienne (diminution des tarifs pour les destinations les plus achalandées)

Quant aux consommateurs, des changements interviennent modifiant ses attitudes et ses valeurs:

- vieillissement de la population;

- fractionnement des vacances;

- recherche du plaisir et de l'exotisme;

- exigence quant au raffinement, à l'animation et à la qualité des services.

Dans l'économie canadienne et québécoise, des modifications vont affecter le tourisme québecoois. Ces changements sont:

- la valeur du dollar canadien et des monnaies étrangères;

- le libre-échange avec les États-Unis;

- la réforme fiscale canadienne;

- la dérégionalisation.

Quant aux constats relevant de l'environnement politique, une volonté de concertation d'une part entre le gouvernement fedéral (Canada) et le gouvernement provincial (Québec) et d'autre part entre les provinces (l'Ontario et le Québec), modifie les relations entre les intervenants gouvernementaux.

Le tableau 1 identifie les menaces et les opportunités du tourisme québécois sous $I^{\text {t}}$ angle de la demande, de l'environnement et de la concurrence.

\section{Le diagnostic}

L'impact économique du tourisme est important dans l'économie québécoise même si en valeur relative son poids a diminué dans le produit intérieur brut $(2,5 \%$ en 1987$)$. Les retombées fiscales sont évaluées à $527 \mathrm{mil}$ lions de dollars pour les deux paliers de gouvernement.

Par contre où le bât blesse, c'est au niveau du différentiel entre les recettes du Québec ( 3 milliards) et les dépenses des Québécois 
(3,5 milliards). Ce defieit de 503 millions en 1986 s'explique d'une part par la désaffection des Québécois pour le Québec el d'autre part par leur intérét soutenu pour les destinations-soleil et leur engouement pour l'outre-mer (exotisme). La part de marché de la destination Québec dans les dépenses des Québécois ne représente plus que $34 \%$ de leurs dépenses.

Le Québec est doté de forces incontestables, que ce soit la nature dans toute sa splendeur, sa spécificité française pour les NordAméricains, le caractère chaleureux des Québécois qui permet une relation visiteur-visité, sa notoriété issue de celle du Canada comme un pays sécuritaire, riche, à conquérir et l'approche associative que le ministère du Tourisme développe avec les entreprises du secteur privé.

Par contre, globalement, le Québec n'a pas suivi l'évolution mondiale des dernières annes. On assiste à un effritement et les causes sont multiples:

- faible conscientisation de l'importance du tourisme comme apport économique, tant de la part du public que des gouvernements;

- offre québécoise dépourvue d'attrait incontestable au niveau international;

- entreprises trop centrées sur elles-mêmes plutôt que sur les exigences du client;

- budgets marketing trop restreints dans une perspective oủ la concurrence accroît ses efforts;

- instances décisionnelles insuffisamment concertées.

Le Québec, face à cet environnement, devra redoubler d'efforts pour présenter des produits compétitifs répondant aux normes internationales les plus exigeantes.

\section{Les objectifs}

D'une part, au niveau qualitatif, un objectif d'efficacité s'impose pour maximiser les retombées économiques. À court terme ceci implique plus un resserrement quant aux choix à faire qu'une volonté systématique d'ouverture.

D'autre part, une approche de "marketingoriented" doit être privilégiée. Cela implique d'être branché sur le processus d'achat du consommateur.

L'approche " 'produit' doit donc être fortement revalorisée par rapport à l'approche "destination globale'. Il faudra intégrer clairement les produits dans le positionnement du Québec. Quant aux objectifs quantitatifs, ils visent une progression des recettes pour Québec plus forte que celles des dépenses. Pour y arriver, il faut accroitre la part de marché du Québec parmi les dépenses des Québecois. Cette part de marché ne représente plus que $34 \%$ des dépenses. La relance de l'intra-Québec est essentielle.
Par ailleurs, il faut une progression des recettes des touristes non-tésidents plus fortes que celle des dépenses des Québécois à l'extêrieur du Québec. Dans un contexte canadien, il faudra que le Québec augmente sa part de marché par rapport aux autres provinces dans les recettes touristiques canadiennes pour atteindre au moins le pourcentage de son poids démographique (26\%). Actuellement, le Québec ne reçoit que $19 \%$ des recettes des touristes d'outre-mer et $18.9 \%$ des dépenses des Canadiens au Canada.

\section{Les axes stratégiques}

Pour atteindre les objectifs fixés, tous les efforts de mise en marché doivent être articulés autour de trois grands axes:

\section{Concentration et renforcement}

Le Québec va devoir s'adresser à des marchés moins éparpillés, moins nombreux mais beaucoup plus precis; ceci veut dire: choix et ciblage.

\section{Concertation et leadership}

Les intervenants en matière de tourisme sont nombreux. Seule une concertation authentique entre ces derniers peut amener une cohésion dans les approches.

\section{Association et alliances}

Sur la scène internationale, il faudra identifier le Québec comme une région du Canada, compte tenu de la faible notoriété du Québec et du phénomène de continentalisation. De plus, il faudra établir des alliances entre les différents paliers de gouvernement, entre l'État et les grandes entreprises qui ont intérêt à ce que les étrangers voyagent au Québec.

\section{Le plan stratégique d'ensemble}

\section{Choix stratégiques concernant \\ les marchés-cibles}

Les stratégies de marketing débutent avec un choix de marchés sur lesquels le Québec conviendra de se positionner.

\section{Les marchés primaires}

Ces marchés se caractérisent par le fait qu'ils contribuent fortement au chiffre d'affaires et que le Québec leur est très facilement accessible. Ces marchés doivent faire l'objet d'une approche "high-profile" (publicité), le Québec doit y étre promu en tant que tel et non comme une composante du Canada. Ces marchés sont

- le Québec (50\% des recettes du tourisme d'agrément),

- l'Ontario (la ville de Toronto),

- le nord-est américain (NouvelleAngleterre et Centre-Atlantique).

\section{Les marchés secondaires}

Ces marchés nécessitent une action promotionnelle moins intensive que les marchés primaires et, dans la mesure du possible, ils devront être développés en association. Ces marches sont:
- les États de l'Atlantique-sud (U.S.A.),

- les États du centre-nord-est (U.S.A.),

- la France.

\section{Les marchés tertiaires}

Ces marchés constituent des segments à potentiel incontestable mais le Quebec n'y a pas encore fait de percée, sa notoriété y étant presque nulle. Le Québec n'y est et n'y sera, dans un horizon prévisible, qu'une région du Canada ou du nord-est de l'Amérique du Nord. Ces marchés sont:

- la Californie (U.S.A.),

- le Japon et l'Allemagne.

- la Grande-Bretagne.

\section{Le positionnement}

Dans un environnement aussi concurrentiel que le tourisme, la différenciation devient la clé-de-voûte pour une destination comme le Québec. Ce positionnement doit s'appuyer sur un avantage différentiel réel, fort, unique, inattaquable dans la mesure du possible. Le Québec doit aussi tenir compte des différents marchés auxquels il s'adresse, il peut se donner plusieurs positionnements à la condition que ceux-ci ne soient pas contradictoires.

\section{Positionnement en Ontario et} aux États-Unis:

la spécificité culturelle et patrimoniale

En tourisme, la différence s'appelle souvent exotisme. Sur le continent nord-américain, le Québec peut être perçu comme exotique en mettant en évidence sa différence culturelle. Ceci ne signifie nullement qua la langue française constitue à elle seule cette différenciation.

Positionnement à l'outre-mer:

l'accessibilité des grands espaces et le fleuve

L'attrait du spectaculaire est souvent à l'origine d'un voyage outre-mer. Le Canada est perçu comme un pays où la nature est fortement présente avec tous les éléments qui s'y rattachent (origines autochtones, trappeurs, animaux sauvages... ). Le Québec doit se différencier des autres en y ajoutant une image qui lui est propre.

\section{Positionnement à l'intra-Québec:} la diversité et le plaisir

Le Québec doit se positionner sans complexe auprès des Québécois. La diversité doit transparaître au niveau des produits, des saisons, des activités qu'il convient de découvrir ou de redécouvrir.

\section{La stratégie du produit}

Le Québec dispose d'un choix de produits extrêmement variés qu'il convient de regrouper en gamme de produits: ces produits ont reçu leur appellation à partir de l'activité principale justifiant le déplacement, ils font référence à une expérience de vacances. 
Les produits de base à fortes retombées économiques sont des produits qui assurent actuellement les $2 / 3$ du chiffre d'affaires du tourisme québécois. Ces produits sont:

- les grandes villes (Montréal, Québec et Hull-Ottawa),

- le ski alpin (9 stations majeures).

- les congrès et réunions de compagnies (retombées économiques importantes).

Les produits de base à relancer sont des produits identifiés au Québec mais actuellement en perte de vitesse:

- le circuit (relance des régions),

- la chasse et la pêche (collées à l'image du Québec),

- le séjour ("resorting") incluant plusieurs activités.

Les produits à développer sont des produits en emergence sous un volet touristique:

- la motoneige (identifiée au Québec),

- l'aventure (produit vitrine appelant le consommateur).

Le Québec devra déployer des efforts pour ces produits prioritaires dans des proportions differrentes selon leur classement au sein du tourisme québécois et selon leur niveau de maturité. Il faudra intégrer dans la mise en marché de ces produits des principes directeurs qui sont les suivants:

- sélectivité,

- regroupement de produits ("packaging"),

- groupe de référence (bannière),

- encouragement au produit hautde-gamme,

- mise en marché des produits substituts actuellement consommés ailleurs,

- renforcement des aspects "acteurs" plutôt que des aspects "spectateurs",

- renforcement de la spécificité culturelle,

- renforcement de l'animation et du service à la clientèle.

\section{La stratégie de mise en marché}

Sous le vocable "mise en marché", il faut inclure l'ensemble des choix à effectuer et des moyens à mettre en oeuvre pour faire en sorte que les clientèles-cibles achètent en tout ou en partic la gamme de produits.

\section{Stratégie de distribution}

Il existe un problème de taille dans la manière de rejoindre le consommateur, celui-ci est très sollicité par des produits concurrents et n'est pas suffisamment informé de l'existence et des avantages des produits québécois. Les agents réceptifs et les grossistes ont besoin de bons produits et d'une bonne promotion auprès du consommateur de la part des entreprises et/ou du ministère pour qu'ils puissent emboiter le pas et récupérer les efforts de communication en combinant transport, hébergement et attraits sous forme de forfaits.

Les grossistes connaissent généralement mieux la demande internationale que les tenants de l'offre, par contre les agents réceptifs connaissent la polyvalence de cette offre. Une concertation et une collaboration des deux permettront de mieux structurer le produit et ainsi de le rendre plus facilement exportable.

\section{Stratégie des prix}

Le Québec n'est pas une destination bon marché et ne le sera jamais. La loi de l'offre et de la demande exerce une pression à la baisse qui a de fâcheuses répercussions sur le niveau des profits, donc sur la rémunération de la main-d'oeuvre et le niveau de qualification de celle-ci

Les entreprises, pour trouver un meilleur rendement et pour plaire aux consommateurs, pourraient appliquer la stratégie du buffet. Cette stratégie consiste à proposer au consommateur une séric d'activités pour un prix forfaitaire inférieur à la somme effective de chacune des composantes puisque le client ne consommera qu'une partie de l'offre.

\section{Stratégie de communication}

La première étape consiste en un éveil de la destination. La publicité conventionnelle avec une image et un slogan fort et des articles dans les journaux constituent les principaux canaux à utiliser pour les marchés primaires. Les produits offerts devront dominer dans ces campagnes de publicité mais seront aussi enrobés dans l'image de la destination globale. Les marchés secondaires et tertiaires utiliseront davantage les relations de presse.

La seconde étape est celle de l'exploration. Les intervenants répondront à cette étape en donnant de l'information à travers les bureaux d'accueil, les renseignements téléphoniques et le courrier. Il est important de s'assurer de l'efficacité dans la réponse et dans le temps d'acheminement de la documentation écrite (le plus court possible).

Les publications, compte tenu des considérations budgétaires, devront être regroupées sous les volets agrément-été, agrément-hiver et affaires. Ces brochures visent d'abord à éveiller, attirer le consommateur sur les produits du Québec, à l'informer sur les possibilités concrètes et à le rassurer. Toutes les publications devront être cohérentes quant au positionnement à l'harmonie visuelle. Un graphique de collection et une signature serviront de trait d'union. Cette signature efficace, simple et, surtout, en relation avec l'avantage différentiel du Québec, devra être utilisée sur les imprimés et sur les autres supports promotionnels.
La force de vente est aussi un ćlé́ment essentiel de la mise en marché. Elle se retrouve sous un premier volet au Québec à travers les bureaux d'information touristique, les centres d'accueil, les maisons régionales et frontalières et sous un volet international par la présence de conseillers en territoire. Le personnel doit être considéré comme des vendeurs: c'est la véritable force de frappe du Québec. Particulièrement pour les conseillèrs en territoire, le démarchage, travail de fourmi, devra être mené auprès des médias, des groupes de clients et grossistes.

\section{Le service à la clientèle}

Le service à la clientèle doit être considéré comme faisant partic des stratégies de mise en marché du tourisme québécois. Le problème de la main-d'ocuvre est présent réside dans le manque de formation.

Ces lacunes se retrouvent tant au niveau des connaissances géographiques, historiques, linguistiques qu'au niveau des connaissances de marketing. La formation est essentielle pour donner un service exceptionnel à la clientèle. Lorsque les produits à offrir, dans une perspective concurrentielle, sont de moindre envergure, le service à la clientêle est un atout majeur pouvant différencier la destination de ses concurrents. Pour améliorer cette qualité d'accueil, tant dans le milieu touristique que dans le grand public, une campagne d'hospitalité devrait être menée afin de sensibiliser les intervenants et la population aux attitudes d'accueil et à I'importance économique du tourisme.

\section{Les implications du plan marketing}

Toutes ces stratégies ont des implications organisationnelles pour les intervenants tant au ministère du Tourisme, dans les associations touristiques régionales que dans les entreprises elles-mêmes. Pour améliorer la situation du Québec, une stratégie de concertation est essentielle.

D'abord, une concertation entre le ministère du Tourisme et les entreprises. L'approche suggérée pour coordonner cette stratégie dans une même direction est double:

- d'une part une approche institutionnelle où le ministère du Tourisme est le seul interlocuteur. Elle a pour objectif de positionner le Québec comme destination globale et de faire connaitre les différents produits à travers une publicité, des publications, des relations de presse, une présence dans des salons, les services d'accueil;

- d'autre part, une approche associative entre le ministère du Tourisme et les intervenants permettra, sous l'initiative du milieu, de stassocier afin d'ajouter d'autres actions marketing auprès du public et des intermédiaires. Les actions tant institutionnelles qu'associatives devront $s$ 'intégrer à des plans de marketing par produit (ski alpin, circuit...). 
TABLEAU 1

Sommaire des menaces et opportunités du tourisme québécois

Variables incontrôlables

\begin{tabular}{|c|c|c|}
\hline Environnement & Menace & Opportunités \\
\hline Demande & $\begin{array}{l}\text { La désaffection des Québécois pour leur prowince } \\
\text { Le goút de voyager des Québécois et le rattrapage à effectuer par } \\
\text { rapport aux autres Canadiens fice aux destinations d'outre-mer } \\
\text { Régression de l'obstacle distance } \\
\text { La recherche de l'exotisme }\end{array}$ & $\begin{array}{l}\text { Perspectives de croissance forte au niveau local et au niveau } \\
\text { international grâce d: } \\
\text { - aceroissement des revenus } \\
\text { - changement dans les valeurs } \\
\text { - facilités de communiquer et de voyager } \\
\text { Le goùt de voyager des Québécois et le rattrapage à effectuer par } \\
\text { rapport aux autres Canadiens } \\
\text { Le vieillissement ("baby-boomers", troisième àge,..) } \\
\text { Les valeurs axées sur le besoin d'aventure et le besoin de sécurite } \\
\text { Le fractionnement des vacances }\end{array}$ \\
\hline Économie: & $\begin{array}{l}\text { Remonté a court terme du dollar canadien par rapport au dollar } \\
\text { américain } \\
\text { La continentalisation } \\
\text { Concentration de l'économie autour des grandes métropoles } \\
\text { La monté de Toronto } \\
\text { La dérégionalisation }\end{array}$ & $\begin{array}{l}\text { Faiblesse du dollar canadien par rapport aux devises europénnes } \\
\text { et japonaise } \\
\text { La prise de conscience dans les regions que le tourisme est une } \\
\text { priorité conomique } \\
\text { La tertiarisation supportera les voyages d'affaires }\end{array}$ \\
\hline Légal & Déréglementation & \\
\hline Politique & $\begin{array}{l}\text { Abandon des politiques de redistribution } \\
\text { Les restrictions budgétaires }\end{array}$ & $\begin{array}{l}\text { Concertation fédérale - provinciale } \\
\text { Concertation Ontario - Québec }\end{array}$ \\
\hline Concurrence & $\begin{array}{l}\text { La force d'attraction de toutes les destinations au climat plus } \\
\text { agréable et/ou au patrimoine plus riche } \\
\text { La stratégie et les moyens financiers de l'Ontario }\end{array}$ & $\begin{array}{l}\text { Association avec les concurrents geographiquement immédiats et } \\
\text { avee Tourisme Canada }\end{array}$ \\
\hline $\begin{array}{l}\text { Événement } \\
\text { conjoncturel }\end{array}$ & & Le 350ème anniversaire de Montréal en 1992 \\
\hline
\end{tabular}

La démarche de concertation qui a été entreprise pour ce mandat devra se renouveler afin de réviser les orientations et les actions pour les adapter le plus rapidement possible aux changements.

Le tourisme est un concept intégrateur et beaucoup d'autres ministeres au sein du gouvernement ont un rôle à jouer par les programmes qu'ils gèrent et qui sont reliés directement ou indirectement au secteur tourisme. La concertation interministérielle déjà amorcée devra devenir une priorité pour cumuler tous les efforts dans le mème sens.

\section{Conclusion}

Au cours des trois prochaines années, le Québec doit rattraper une partie de son retard et pour cela la relance de l'intra-Québec est essentielle. La clientèle internationale est importante mais se fier trop exclusivement à elle (compte tenu des éléments conjoncturels) est un risque trop grand dans une situation où le bilan des recettes et dépenses est largement déficitaire.
Les axes de concentration et de renforcement exigent des choix difficiles à faire mais à court terme ils sont primordiaux. Lorsque la situation aura pris une tangente positive, une ouverture sur des marchés nouveaux en alliance avec d'autres partenaires sera possible. De plus, cette nouvelle approche produit dans un contexte de destination globale (le Québec) rếpond à des besoins du consommateur. Ce dernier veut vivre une expérience et c'est en lui présentant une gamme de produits qu'il pourra plus facilement associer des activités à une destination.

Cette étude, qui a été menée dans un délai très court, a quand même permis de faire le point, non pas exclusivement sur une planification marketing mais d'amorcer une réflexion plus globale sur les orientations stratégiques du tourisme québécois et cela en collaboration avec les intervenants.

Ce travail a permis de mieux situer le potentiel des différents produits du Québec et de voir dans une perspective concurrentielle les forces et les faiblesses de chacun, permettant ainsi de prendre un recul essentiel pour juger des actions à entreprendre. 